\title{
Hipotiroidismo Associado a Anticorpos Anti-Receptor da Hormona TSH com Ação Bloqueadora Determinada In Vitro
}

\author{
Hypothyroidism Associated to TSH Hormone-Receptor Autoantibodies with \\ Blocking Activity Assessed In Vitro
}

Pedro MARQUES ${ }^{1}$, Karim CHIKH², Anne CHARRIÉ², Rosa PINA³, Maria João BUGALHO ${ }^{1}$, Lurdes LOPES ${ }^{3}$

Acta Med Port 2015 Sep-Oct;28(5):663-666

\section{RESUMO}

Os anticorpos anti-receptor da hormona estimulante da tiróide causam habitualmente hipertiroidismo. No entanto, a sua atividade pode ser bloqueadora, condicionando o hipotiroidismo. Apresenta-se o caso de uma doente do sexo feminino, 11 anos, com diabetes mellitus tipo 1, doença celíaca e tiroidite linfocítica em eutiroidismo ao diagnóstico. Cerca de dois anos após a avaliação inicial, a hormona estimulante da tiróide apresentava-se suprimida com T4 livre normal. Nove meses depois, a evolução foi para hipotiroidismo notandose concomitantemente elevação dos anticorpos anti-receptor da hormona estimulante da tiróide, mantendo-se a doente sempre assintomática. Células chinese hamster ovary foram transfetadas com o receptor de hormona estimulante da tiróide, e após incubação com o soro da doente, verificou-se uma atividade bloqueadora 'moderada' dos anticorpos anti-receptor da hormona estimulante da tiróide, excluindo-se ação estimuladora concomitante. Neste caso, o reconhecimento da ação bloqueadora dos anticorpos anti-receptor da hormona estimulante da tiróide detetados no soro suporta a hipótese de uma etiologia multifatorial para o hipotiroidismo que, na ausência dos testes in vitro, tenderíamos a interpretar unicamente como sequela do processo destrutivo associado à tiroidite linfocítica.

Palavras-chave: Doença de Graves; Hipotiroidismo; Receptores da Tireotropina; Tireoidite Autoimune.

\section{ABSTRACT}

Thyroid-stimulating hormone-receptor autoantibodies normally causes hyperthyroidism. However, they might have blocking activity causing hypothyroidism. A 11-year-old girl followed due to type 1 diabetes mellitus, celiac disease and euthyroid lymphocytic thyroiditis at diagnosis. Two years after the initial evaluation, thyroid-stimulating hormone was suppressed with normal free T4; nine months later, a biochemical evolution to hypothyroidism with thyroid-stimulating hormone-receptor autoantibodies elevation was seen; the patient remained always asymptomatic. Chinese hamster ovary cells were transfected with the recombinant human thyroid-stimulating hormone -receptor, and then exposed to the patient's serum; it was estimated a 'moderate' blocking activity of these thyroid-stimulating hormone-receptor autoantibodies, and concomitantly excluded stimulating action. In this case, the acknowledgment of the blocking activity of the serum thyroid-stimulating hormone-receptor autoantibodies, supported the hypothesis of a multifactorial aetiology of the hypothyroidism, which in the absence of the in vitro tests, we would consider only as a consequence of the destructive process associated to lymphocytic thyroiditis.

Keywords: Graves Disease; Hypothyroidism; Receptors, Thyrotropin; Thyroiditis, Autoimmune.

\section{INTRODUÇÃO}

Os anticorpos anti-receptor da hormona estimulante da tiróide (TRAbs) ao ligarem-se a este receptor têm, habitualmente, uma ação similar à da hormona estimulante da tiróide (TSH) conduzindo à produção de hormonas tiroideias. Os TRAbs são causa direta da doença de Graves (DG), a principal forma de hipertiroidismo. ${ }^{1}$ Raramente, a ligação dos TRAbs aos receptores da TSH não só não promove transdução de sinal como bloqueia a ação da TSH favorecendo evolução para hipotiroidismo. ${ }^{2}$

A atividade dos TRAbs pode ser estimada in vitro através de bioensaios. Atualmente, os bioensaios mais sofisticados empregam células chinese hamster ovary $(\mathrm{CHO})$ transfetadas com receptores de TSH; a atividade biológica é deduzida a partir da adenosina 3',5'-monofosfato (AMP) cíclico produzida. ${ }^{3,4}$ Estes bioensaios estão só disponíveis em laboratórios diferenciados, e apesar da sua utilidade clínica ser controversa e limitada, podem constituir um contributo importante para a compreensão de casos particulares de natureza autoimune..$^{5,6}$

Reporta-se o caso de uma doente com hipotiroidismo associado a TRAbs com ação bloqueadora, esta demonstrada através de testes in vitro.

\section{CASO CLÍNICO}

Doente do sexo feminino, 11 anos, seguida regularmente em consulta, com os diagnósticos de diabetes mellitus tipo 1, doença celíaca e tiroidite linfocítica (TL) em eutiroidismo. O diagnóstico desta última entidade assentou na documentação de anticorpos anti-peroxidase tiroideia (antiTPO) e anti-tiroglobulina (AATg) positivos e numa ecografia sugestiva de TL (tiróide de dimensões ligeiramente reduzidas e com ecoestrutura francamente heterogénea). Sem história familiar de doença tiroideia. Ao longo do seguimento, cerca de dois anos após o diagnóstico da diabetes e da doença celíaca, observou-se uma TSH suprimida com T4 livre normal (Tabela 1); posteriormente, assistiu-se

1. Serviço de Endocrinologia. Instituto Português de Oncologia Dr. Francisco Gentil. Lisboa. Portugal.

2. Departamento de Bioquímica. CARMEN Laboratory. University Claude Bernard Lyon. Lyon Sud Hospital. Hospices Civils de Lyon. Lyon. França.

3. Serviço de Endocrinologia Pediátrica. Hospital Dona Estefânia. Centro Hospitalar de Lisboa Central. Lisboa. Portugal.

$\square$ Autor correspondente: Pedro Marques. pedro.miguel.sousa.marques@gmail.com

Recebido: 15 de Abril de 2015 - Aceite: 01 de Junho de 2015| Copyright @ Ordem dos Médicos 2015 
Tabela 1 - Resultados analíticos da doente e a sua evolução ao longo do tempo

\begin{tabular}{|c|c|c|c|c|c|}
\hline & Outubro 2013 & Março 2014 & Julho 2014 & Outubro 2014 & $\begin{array}{l}\text { Valores de } \\
\text { referência }\end{array}$ \\
\hline TSH & 0,027 & 0,870 & 12,651 & $2,614^{*}$ & $0,3-2,9 \mu \mathrm{UI} / \mathrm{mL}$ \\
\hline T4 livre & 1,00 & 0,86 & 0,56 & $0,67^{*}$ & $0,68-1,05 \mathrm{ng} / \mathrm{dL}$ \\
\hline T3 livre & & 4,75 & & $3,58^{*}$ & $2,6-5,7 \mathrm{pg} / \mathrm{mL}$ \\
\hline TRAbs & & 30,3 & $26,3^{\dagger}$ & & $<1,0 \mathrm{UI} / \mathrm{L}$ \\
\hline Anti-TPO & 93,8 & 161,2 & & 379,2 & $<9,0 \mathrm{UI} / \mathrm{mL}$ \\
\hline AATg & 14,5 & 67,9 & & 54,1 & $<4,0 \mathrm{UI} / \mathrm{mL}$ \\
\hline
\end{tabular}

* Resultados sob levotiroxina, iniciada perante a avaliação analítica de $26 / 07 / 14 .{ }^{\dagger}$ Amostra na qual foi analisada a atividade biológica dos TRAbs

à evolução rápida para hipotiroidismo com título elevado de TRAbs (Tabela 1), motivando o início de terapêutica com levotiroxina. Apesar das oscilações de função tiroideia, a doente manteve-se sempre assintomática.

Uma amostra de soro da doente foi enviada para o Hospital de Lyon com o intuito de estimar a atividade biológica dos TRAbs e definir o seu papel no hipotiroidismo desta doente. Células $\mathrm{CHO}$ foram transfetadas com o receptor de TSH recombinante humano, e após incubação com o soro da doente, a atividade dos TRAbs foi estimada em função do AMP cíclico produzido (Tabela 2). ${ }^{4}$ Foi identificada uma atividade bloqueadora dos TRAbs estimada em $26 \%$ (atividade bloqueadora moderada), excluindo-se concomitantemente qualquer atividade estimuladora associada aos TRAbs (Tabela 2; Fig. 1). Concluiu-se que a atividade bloqueadora dos TRAbs nesta doente possa ter contribuído para a evolução para hipotiroidismo, isoladamente ou associada à evolução natural da TL.

\section{DISCUSSÃO}

Neste caso o estudo da atividade biológica dos TRAbs demonstrou uma incomum e importante ação bloqueadora. O hipotiroidismo nesta doente pode ter resultado da ativi-

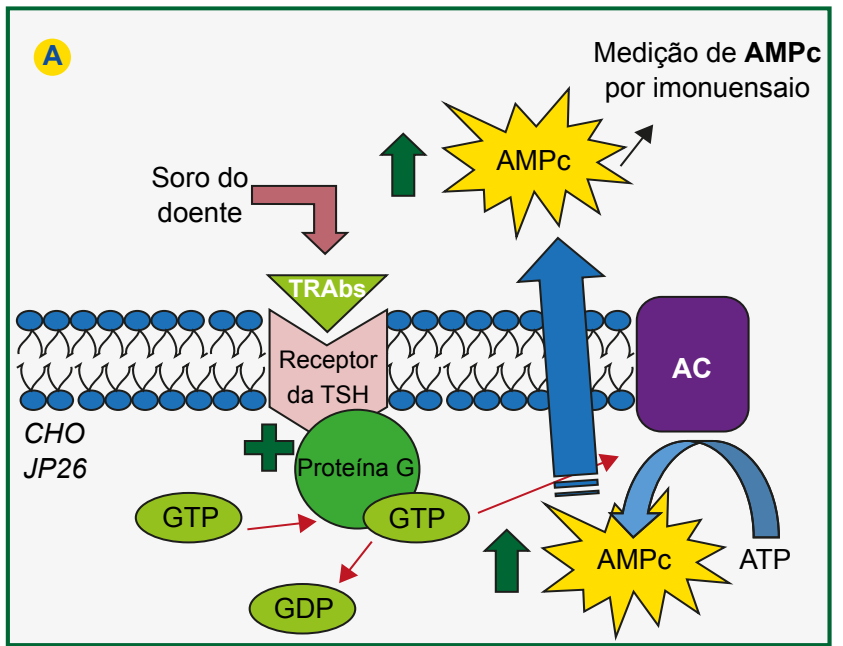

dade bloqueadora dos TRAbs isoladamente ou em associação com os efeitos dos antiTPO e AATg. A ausência de atividade estimuladora, excluiu a coexistência de TRAbs estimuladores e bloqueadores. ${ }^{2}$ Nestes casos, o tipo de disfunção tiroideia resultante é determinada pelas concentrações relativas de cada fração de TRAbs, bem como da sua afinidade relativa para o receptor da $\mathrm{TSH}_{.}$, 2,6-8

A evolução de tirotoxicose/hipertiroidismo subclínico para hipotiroidismo clínico pode enquadrar-se simplesmente na história natural da TL, cujo processo destrutivo autoimune condiciona libertação das hormonas tiroideias (tirotoxicose transitória), e posterior hipotiroidismo inerente à destruição glandular e consequente diminuição da reserva funcional da tiróide. A este processo, no entanto, pode-se ter associado a ação bloqueadora dos TRAbs. Alternativamente, esta oscilação entre hipertiroidismo e hipotiroidismo pode ter ocorrido por alteração da natureza/atividade biológica dos TRAbs, uma situação infrequente mas descrita na literatura. ${ }^{2,9}$ Dado que o estudo dos TRAbs foi realizado somente na fase de hipotiroidismo, não se pode excluir definitivamente a ocorrência deste fenómeno.

Este caso exemplifica o quão difícil pode ser a definição

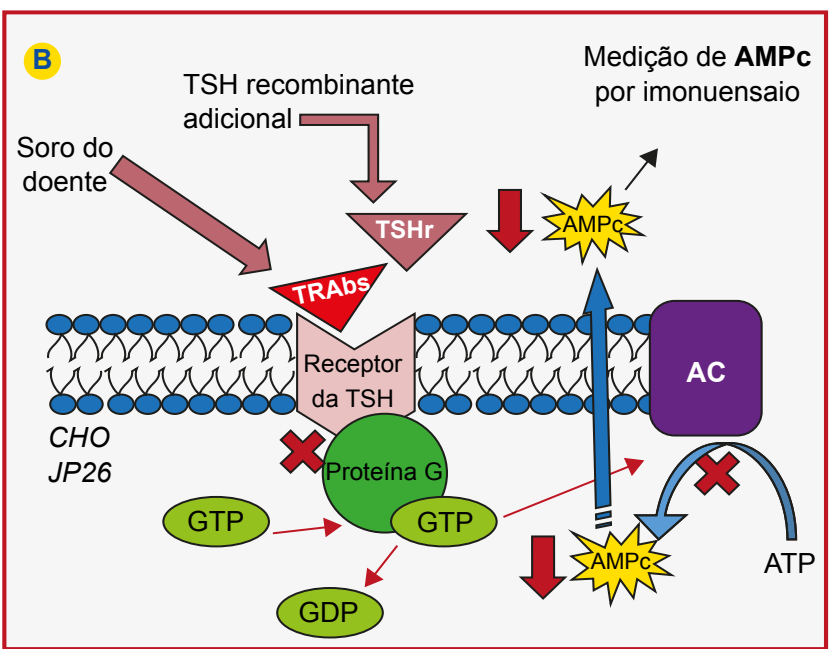

Figura 1 - Esquema ilustrativo do método de determinação in vitro da atividade estimuladora (A) e bloqueadora (B) dos receptores da TSH pelos TRAbs 
Tabela 2 - Descrição do bioensaio usado para determinação da atividade biológica dos TRAbs ${ }^{4}$

Local do bioensaio Departamento de Biologia, Hospices Civils de Lyon Sud University Hospital

Procedimentos técnicos
- células CHO são transfetadas com o receptor de TSH recombinante humano;

- as células CHO JP-26 são colocadas em 96 placas (50 000 células por placa), cultivadas em soro bovino Ham's F12 Nutrient Medium e usadas para os bioensaios após um período de 24 horas;

- as células CHO são depois expostas por 2 horas a $4 \mu \mathrm{L}$ de soro teste ou $196 \mu \mathrm{L}$ de soro controlo, suplementados com $10 \mathrm{mM}$ de HEPES, $0,25 \mathrm{mmol} / \mathrm{l}$ de isobutilmetilxantina e $0,75 \%$ de albumina sérica bovina (ASB) $\mathrm{pH} 7,4$;

- nos bioensaios para determinação da atividade bloqueadora dos TRAbs é adicionado $0,1 \mathrm{mIU} / \mathrm{mL}$ de TSH recombinante;

- após a incubação com o soro da doente (e controlos), o AMP cíclico libertado pelas células é quantificado (método radioimunoensaio para determinação do AMP cíclico; IMMUNOTECH, a Beckman Coulter Company, Marseille, France).

- soros controlos sem TRAbs ('soros normais') são usados para medir o AMP cíclico produzido em condições basais ('normais'); soros controlo com TRAbs estimuladores são usados como controlos positivos nos bioensaios de atividade estimuladora; soros controlo com TRAbs bloqueadores são usados como controlos positivos nos bioensaios de atividade bloqueadora.
Aferição I

quantificação da

atividade biológica dos TRAbs
- a atividade estimuladora dos TRAbs é definida/classificada por comparação com a percentagem de AMP cíclico produzida em condições basais:

\# 140 - 200\%: atividade estimuladora dos TRAbs 'nula ou baixa'

\# 200 - 400\%: atividade estimuladora dos TRAbs 'moderada'

\# > 400\%: atividade estimuladora dos TRAbs 'intensa'

- a atividade bloqueadora dos TRAbs é calculada pela fórmula 1 - (a/b) x 100, em que:

a representa o AMPc gerado na presença da amostra de soro do doente + TSH recombinante humana adicionada

b representa o AMPc gerado na presença de um soro controlo 'normal' + TSH recombinante humana adicionada

\# 10 - 20\%: atividade bloqueadora dos TRAbs 'baixa'

\# 20 - 40\%: atividade bloqueadora dos TRAbs 'moderada'

\# > 40\%: atividade bloqueadora dos TRAbs 'intensa'

- os bioensaios são feitos em triplicado; os resultados são calculados e apresentados como a média resultante dos três ensaios.

- na atividade estimuladora dos TRAbs: 8,6\%

- na atividade bloqueadora dos TRAbs: 7,1\%
Variabilidade
inter-bioensaios

de fronteiras entre DG e TL e a controvérsia que este assunto tem gerado. Apesar de tradicionalmente serem consideradas como duas patologias distintas, vários aspetos têm levantado a hipótese destas entidades serem espectros opostos da mesma doença. ${ }^{10,11}$ Além da infiltração linfocitária e presença de AATg e antiTPO na DG e TL, outros fatores suportam esta hipótese: a formação de TRAbs em ambas as condições (apesar de resultados biológicos distintos), ${ }^{2}$ a interconversão entre $\mathrm{DG}$ e $\mathrm{TL}^{10,12}$; a observação, na mesma família, de elementos com DG e outros com $\mathrm{TL}^{13}$ Os bioensaios dos TRAbs podem contribuir para a evolução do conhecimento a este nível.

Duas importantes limitações são reconhecidas aos bioensaios: primeiro, a atividade bloqueadora dos TRAbs é calculada em função da percentagem de inibição da atividade em relação a um controlo com baixa concentração de TSH, o que por si só pode subestimar a atividade bloqueadora dos TRAbs. A segunda limitação é essencialmente conceptual e prende-se com a classificação dos TRAbs: frequentemente os TRAbs com atividade bloqueadora são designados como 'bloqueadores', quando na verdade têm atividade estimuladora, mas de menor intensidade comparativamente à $\mathrm{TSH}$, funcionando como agonistas parciais fracos. ${ }^{2,14}$

A definição da atividade biológica dos TRAbs é exequível em laboratórios dedicados ao estudo de doenças autoimunes em particular da tiróide. Importa salientar que as decisões terapêuticas devem ser baseadas na avaliação clínica e da função tiroideia, independentemente dos resultados dos bioensaios dos TRAbs. ${ }^{2}$ Na prática clínica, pode haver interesse neste tipo de estudos em situações específicas tais como: doentes com função tiroideia lábil (oscilações frequentes entre hipotiroidismo/hipertiroidismo); oftalmopatia de Graves em eutiroidismo/hipotiroidismo; tiroidite pós-parto; DG na gravidez; hipotiroidismo ou hipertiroidismo neonatal. ${ }^{2,4}$

Neste caso, enquadrável na síndrome poliglandular autoimune tipo $\| I^{15}$, o reconhecimento da atividade blo- 
queadora dos TRAbs facilitou a interpretação da disfunção tiroideia de natureza autoimune, permitindo admitir a hipótese de uma etiologia multifatorial para o hipotiroidismo que, na ausência dos testes in vitro, tenderíamos a interpretar unicamente como sequela do processo destrutivo associado à $\mathrm{TL}$.

\section{PROTECÇÃO DE PESSOAS E ANIMAIS}

Os autores declaram que os procedimentos seguidos estavam de acordo com os regulamentos estabelecidos pelos responsáveis da Comissão de Investigação Clínica e Ética e de acordo com a Declaração de Helsínquia da Associação Médica Mundial.

\section{REFERÊNCIAS}

1. Rapoport B, Chazenbalk GD, Jaume JC, McLachlan SM. The thyrotropin (TSH) receptor: interaction with TSH and autoantibodies. Endocr Rev. 1998;19:673-716.

2. McLachlan SM, Rapoport B. Thyrotropin-blocking autoantibodies and thyroid-stimulating autoantibodies: potential mechanisms involved in the pendulum swinging from hypothyroidism to hyperthyroidism or vice versa. Thyroid. 2013;23:14-24

3. Hinds WE, Takai N, Rapoport B, Filetti S, Clark OH. Thyroid-stimulating immunoglobulin bioassay using cultured human thyroid cells. J Clin Endocrinol Metab. 1981;52:1204-10.

4. Payrat JA, Chikh K, Bossard N, Bretones P, Gaucherand P, Claris $\mathrm{O}$, et al. Predictive value of maternal second-generation thyroidbinding inhibitory immunoglobulin assay for neonatal autoimmune hyperthyroidism. Eur J Endocrinol. 2014;171;451-60.

5. Takasu N, Matsushita M. Changes of TSH-stimulation blocking antibody (TSBAb) and thyroid-stimulating antibody (TSAb) over 10 years in 34 TSBAb-positive patients with hypothyroidism and in 98 TSAb-positive Graves' patients with hyperthyroidism: reevaluation of TSBAb and TSAb in TSH-receptor-antibody (TRAb)-positive patients. J Thyroid Res. 2012;182176.

6. Morgenthaler NG, Ho SC, Minich WB. Stimulating and blocking thyroidstimulating hormone (TSH) receptor autoantibodies from patients with Graves'disease and autoimmune hypothyroidism have very similar concentration, TSH receptor affinity and binding sites. J Clin Endocrinol Metab. 2007;92:1058-65.

7. Takasu N, Yamada T, Takasu M, Komiya I, Nagasawa Y, Asawa T, et al. Disappearance of thyrotropin-blocking antibodies and spontaneous recovery from hypothyroidism in autoimmune thyroiditis. N Engl J Med. 1992;326:513-8

\section{CONFIDENCIALIDADE DOS DADOS}

Os autores declaram ter seguido os protocolos do seu centro de trabalho acerca da publicação dos dados de doentes.

\section{CONFLITOS DE INTERESSE}

Os autores declaram não haver qualquer conflito de interesse.

\section{FONTES DE FINANCIAMENTO}

Não foi recebido qualquer subsídio ou bolsa.

8. Evans M, Sanders J, Tagami T, Sanders P, Young S, Roberts E, et al Monoclonal autoantibodies to the TSH receptor, one with stimulating activity and one with blocking activity, obtained from the same blood sample. Clin Endocrinol. 2010;73:404-12.

9. Khoo DH, Eng $\mathrm{PH}$, Ho SC, Fok AC. Differences in the levels of TSH-binding inhibitor immunoglobulins in goitrous and agoitrous autoimmune thyroiditis after twelve months of L-thyroxine therapy. Clin Endocrinol. 1999;51:73-9.

10. McLachlan SM, Nagayama Y, Pichurin PN, Mizutori Y, Chen C, Misharin A, et al. The link between Graves' disease and Hashimoto's Thyroiditis: a role for regulatory T cells. Endocrinology. 2007;148:5724-33.

11. Wang S, Baker J. The role of apoptosis in thyroid autoimmunity. Thyroid. 2007;17:975-9.

12. Champion B, Gopinath B, Ma G, El-Kaissi S, Wall JR. Conversion to Graves' hyperthyroidism in a patient with hypothyroidism due to Hashimoto's Thyroiditis documented by real-time thyroid ultrasonography. Thyroid. 2008;18:1135-7.

13. Manji N, Carr-Smith JD, Boelaert K, Allahabadia A, Armitage M, Chatterjee VK, et al. Influences of Age, Gender, Smoking, and Family History on Autoimmune Thyroid Disease Phenotype. J Clin Endocrinol Metab. 2006;91:4873-80.

14. Van Sande J, Swillens S, Gerard C, Allgeier A, Massart C, Vassart G, et al. In Chinese hamster ovary K1 cells dog and human thyrotropin receptors activate both the cyclic AMP and the phosphatidylinositol 4,5-bisphosphate cascades in the presence of thyrotropin and the cyclic AMP cascade in its absence. Eur J Biochem. 1995;229:338-43.

15. Ben-Skowronek I, Michalczyk A, Piekarski R, Wysocka-Lukasik B, Banecka B. Type III Polyglandular Autoimmune Syndromes in children with type 1 diabetes mellitus. Ann Agric Environ Med. 2013;20:140-6. 
Pedro MARQUES, Karim CHIKH, Anne CHARRIÉ, Rosa PINA, Maria João BUGALHO, Lurdes LOPES

\section{Hipotiroidismo Associado a Anticorpos Anti-Receptor da Hormona TSH com Ação Bloqueadora Determinada In Vitro}

Acta Med Port 2015:28:663-666

Publicado pela Acta Médica Portuguesa, a Revista Científica da Ordem dos Médicos

Av. Almirante Gago Coutinho, 151

1749-084 Lisboa, Portugal.

Tel: +351218428 215

E-mail: submissao@actamedicaportuguesa.com

www.actamedicaportuguesa.com

ISSN:0870-399X | e-ISSN: 1646-0758

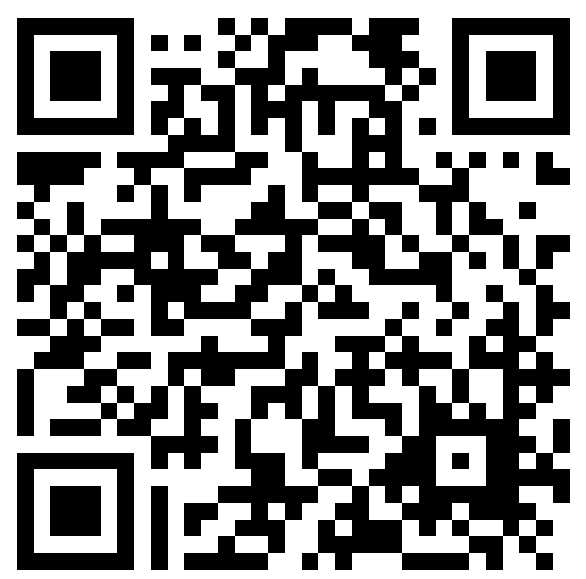

